\title{
Primary Myelofibrosis
}

National Cancer Institute

\section{Source}

National Cancer Institute. Primary Myelofibrosis. NCI Thesaurus. Code C2862.

A chronic myeloproliferative neoplasm characterized by bone marrow fibrosis,

proliferation of atypical megakaryocytes and granulocytes in the bone marrow, anemia, splenomegaly, and extramedullary hematopoiesis. (WHO, 2001) 